\title{
Exact variational representations for the solution of the simple parabolic equation
}

\author{
R.S. Anderssen
}

\begin{abstract}
By constructing a special set of A-orthonormal functions, it is shown that, under certain smoothness as sumptions, the variational and Fourier series representations for the solution of first initial boundary value problems for the simple parabolic differential equation coincide. This result is then extended in order to construct a variational representation for the solution of a very general first initial boundary value problem for this equation.
\end{abstract}

\section{Introduction}

In this paper, we use the results of [1] to investigate the construction of variational representations of the solutions of first initial boundary value problems for the simple parabolic equation:

$$
-\kappa \frac{\partial^{2} u}{\partial x^{2}}+\frac{\partial u}{\partial t}=f(x, t), f \in L_{2}(S(0,1))
$$

$$
u(x, 0)=u(0, t)=u(1, t)=0,
$$

where $K$ is a positive constant, $S(0,1)=\{(x, ' t) ; 0 \leq x \leq 1, t \geq 0\}$ and $L_{2}(S(0,1))$ is the space of real square summable functions defined on $S(0,1)$ with inner product denoted by $($,$) . It follows from [1,52$, Example], that the solution of (1) can be sought as the function which minimises the functional

$$
F_{A}(u)=(A u, A u)-2(A u, f),
$$

Received 14 May 1971. 
where we have identified $A$ with the operator, defined by (1), which maps from the classical solutions of (1) into $L_{2}(S(0,1))$.

We start by constructing special systems of A-orthonormal functions $\left\{\phi_{i j}\right\}=\left\{\phi_{i j}(x, t)\right\} \quad(i=1,2, \ldots ; j=0,1,2, \ldots) \quad$ (see Mikhlin [4; §83]): $\left(A \phi_{i j}, A \phi_{l m}\right)=\delta_{i \tau_{j m}}$ for all $i, j, l$ and $m$ where $\delta_{i j}$ is the Kronecker delta symbol. These functions are used to show that, under certain smoothness assumptions, the Fourier series and the variational representations of the solution of

$$
-\kappa \frac{\partial^{2} v}{\partial x^{2}}+\frac{\partial v}{\partial t}=0
$$

$$
v(x, 0)=F(x), v(0, t)=g(t), v(1, t)=h(t),
$$

coincide, where we define a variational representation of the solution of (3) and (4) by

$$
v=v(x, t)=\sum_{i, j=1}^{\infty} a_{i j} \phi_{i j}+w(x, t),
$$

where $w(x, t)$ is some function which satisfies the initial and boundary conditions (4) and $\sum_{i, j=1}^{\infty} a_{i j} \phi_{i j}=u$ is the variational representation of the function which minimises (2), and hence, of the solution of (1), with $f=\kappa \frac{\partial^{2} w}{\partial x^{2}}-\frac{\partial w}{\partial t}$

If $F(x), g(t)$ and $h(t)$ of (4) are such that:

(a) $F(0)=g(0), F(1)=h(0)$,

(b) $\lim _{t \rightarrow \infty} g(t)=K_{1}, \lim _{t \rightarrow \infty} h(t)=K_{2}$,

(c) $g(t), h(t) \in C^{1}([0, \infty))$ and $F(x) \in C^{2}([0,1])$, and

(d) (1-x) $\frac{\partial g(t)}{\partial t}, x \frac{\partial h(t)}{\partial t}, F(x) e^{-\gamma t}, \frac{\partial^{2} F}{\partial x^{2}} e^{-\gamma t} \in L_{2}(S(0,1))$ for

$$
\gamma>0 \text {, }
$$

then a suitable form for $w$ is

$$
w=w(x, t)=U_{s}(x)+B(x, t),
$$


where $U_{s}(x)=\left(K_{2}-K_{1}\right) x+K_{1}$ denotes the steady state solution of $(3)$ and (4), and

$$
B(x, t)=(1-x)\left\{g(t)-K_{1}\right\}+x\left\{h(t)-K_{2}\right\}+\{F(x)-(1-x) g(0)-x h(0)\} e^{-\gamma t} .
$$

The corresponding form for $f(x, t)$ is

(7) $f(x, t)=-\left[(1-x) \frac{\partial g}{\partial t}+x \frac{\partial h}{\partial t}-\left\{\kappa \frac{\partial^{2} F}{\partial x^{2}}+\gamma\{F(x)-(1-x) g(0)-x h(0)\}\right\} e^{-\gamma t}\right]$.

Condition (a) ensures the initial and boundary conditions match at the corners of $S(0,1)$ and will be referred to as the comer condition; while (b) ensures that the steady state solution of ( 1 ) is zero - a fact required in proof of dense invertibility in [1]. That $f(x, t)$ exists and is contained in $L_{2}(S(0,1))$ follows from (c) and (d).

Finally, we note that it follows from Mikhlin [4, 583] and Petryshyn $[5, \xi 2.1]$ that the $a_{i j}$ in the above variational representation of $u$ are defined by

$$
\sum_{i=1}^{\infty} \sum_{j=0}^{\infty} a_{i j}\left(A \phi_{i j}, A \phi_{m n}\right)=\left(f, A \phi_{m n}\right) \quad(m=1,2, \ldots ; n=0,1,2, \ldots),
$$

which becomes, on using the A-orthonormality of the $\phi_{i j}(x, t)$,

$$
a_{i j}=\left(f, A \phi_{i j}\right) \quad(i=1,2, \ldots ; j=0,1,2, \ldots) .
$$

\section{On the construction of A-orthonormal systems}

Let the A-orthonormal system $\left\{\phi_{i j}\right\}$ be chosen in the form

$$
\phi_{i j}=\phi_{i j}(x, t)=\sqrt{2} \sin (i \pi x) \psi_{i j}(t) \quad(i=1,2, \ldots ; j=0,1,2, \ldots)
$$

with the $\psi_{i j}(t)$ unknown functions of $t$ which satisfy the boundary conditions

$$
\psi_{i j}(0)=0, \quad(i=1,2, \ldots ; j=0,1,2, \ldots) .
$$

It follows from the definition of A-orthonormality that the $\left\{\phi_{i j}\right\}$ are A-orthonormal if the system 


$$
\left\{A \phi_{i j}\right\}=\left\{\sqrt{2} \kappa i^{2} \pi^{2} \sin (i \pi x) \psi_{i j}(t)+\sqrt{2} \sin (i \pi x) \dot{\psi}_{i j}\right\},
$$

where the dot denotes differentiation with respect to $t$, is orthonormal in $L_{2}(S(0,1))$. Such a system is

$$
\left\{\sqrt{2} \sin (i \pi x) \frac{(2 \alpha)^{\frac{1}{2}}}{j !} e^{-\alpha t} L_{j}(2 \alpha t)\right\},
$$

where the $L_{j}(u)$ are the Laguerre polynomials of order $j$. Equating the right hand side of (10) with (11), we obtain

(12) $K \psi_{i j}+\dot{\psi}_{i j}=\frac{(2 \alpha)^{\frac{1}{2}}}{j !} e^{-\alpha t} L_{j}(2 \alpha t) \quad(i=1,2, \ldots ; j=0,1,2, \ldots)$

with $K=\kappa i^{2} \pi^{2}$, which with (9) define the $\psi_{i j}(t)$.

A particular integral of (12) is

$$
C e^{-K t} \int_{0}^{t} L_{j}(2 \alpha u) e^{(K-a) u} d u=\Delta(K, \alpha, j ; t),
$$

with $C=(2 \alpha)^{\frac{1}{2}} / j !$, which can be evaluated using the convolution theorem

$$
\int_{0}^{t} f(u) g(t-u) d u=L^{-I}\{L(f(u)) \cdot L(g(u))\},
$$

where $L$ and $L^{-1}$ denote the Laplace transform and its inverse, and $f(u)=L_{j}(2 \alpha u)$ and $g(u)=e^{-(K-a) u}$.

Since, using $[3, \S \$ 4.11(25), 4.5(1)]$,

$$
L\left(L_{j}(2 \alpha u)\right\}=(p-2 \alpha)^{j} p^{-j-1},
$$

where $\operatorname{real}\{p / 2 \alpha\}>-1$, and

$$
L\left(e^{-(K-\alpha) u}\right)=(p+K-\alpha)^{-1},
$$

where realp $>\operatorname{real}(-K+\alpha)$ and $\alpha \neq k i^{2} \pi^{2}$, we obtain, with $a=K-\alpha$ and realp $>-1$,

$$
\Delta(K, \alpha, j ; t)=C e^{-\alpha t} \sum_{k=0}^{j}(-1)^{k}\left(\begin{array}{l}
j \\
k
\end{array}\right)(2 \alpha)^{k} L^{-1}\left(\left[p^{k+1}(p+a)\right]^{-1}\right) .
$$


Agrin, from $[3, \$ 5.2(21)]$, it is found that

$$
L^{-1}\left(\left[p^{k+1}(p+a)\right]^{-1}\right)=\sum_{l=1}^{k+1}\left\{\frac{(-1)^{l-1}}{(k+1-2) ! a^{2}} t^{k+1-2}\right\}+\frac{e^{-a t}}{a^{k+1}},
$$

whence,

$$
\begin{aligned}
& \text { (13) } \Delta(K, \alpha, j ; t)=\sum_{k=0}^{j}(-1)^{k} \frac{(2 \alpha)^{k+\frac{1}{2}}}{k !(j-k) !} e^{-\alpha t} \sum_{i=1}^{k+1}\left\{\frac{(-1)^{2-1}}{(k+1-l) ! \alpha^{l}}\right. \\
& \left.\left.\times t^{k+1-2}\right\}+\frac{e^{-a t}}{a^{k+1}}\right\}
\end{aligned}
$$

Hence, finally, the complete solution of (9) and (12) is

$$
\psi_{i j}(t)=\Delta(K, \alpha, j ; t)-\Delta(K, \alpha, j ; 0) e^{-K t}
$$

with $K=k i^{2} \pi^{2}, a=K-\alpha$ and $a \neq K$.

Note. The restriction $\alpha \neq k i^{2} \pi^{2}$, introduced earlier, can be removed. When $\alpha \approx k q^{2} \pi^{2}$ ( $q$ an integer), say, one finds, on using [3, $55.2(16)]$, that

$$
\psi_{q j}(t)=\sum_{k=0}^{j}(-1)^{k} \frac{\left(2 k q^{2} \pi^{2}\right)^{k+\frac{1}{2}}}{k !(j-k) !} \frac{t^{k+1} e^{-k q^{2} \pi^{2} t}}{(k+1) !}
$$

3. Exact variational representations of the solution of (1) If $f(x, t)$ is defined by (7), then the $a_{i j}$ of (8) become

$$
\begin{aligned}
a_{i j}=\frac{2 \alpha^{\frac{1}{2}}}{j !}\left\{-\frac{2}{i \pi}\right. & {\left[(-1)^{i}-\frac{1}{2}\right] \int_{0}^{\infty} \frac{\partial g}{\partial t} L_{j}(2 \alpha t) e^{-\alpha t} d t+} \\
& \left.+\frac{(-1)^{i}}{i \pi} \sqrt{2} \int_{0}^{\infty} \frac{\partial h}{\partial t} L_{j}(2 \alpha t) e^{-\alpha t} d t\right\}+\frac{2(y-\alpha)^{j} a^{\frac{1}{2}}}{j !(\gamma+\alpha)^{j+1}} \cdot \\
& \cdot\left\{\int_{0}^{1}\left[\gamma\{F(x)-(1-x) g(0)-x h(0)\}+\kappa \frac{\partial^{2} E}{\partial x^{2}}\right] \sin (i \pi x) d x\right\}
\end{aligned}
$$

where $[3,54.11(31)]$ has been used.

EXAMPLE. Consider (3) and (4) with

$$
E(x)=\sin (\pi x), \quad g(t)=h(t)=0 .
$$

For this initial boundary value problem, 


$$
U_{8}(x)=0, B(x, t)=\sin (\pi x) e^{-\gamma t}, f(x, t)=(\gamma-\kappa \pi) \sin (\pi x) e^{-\gamma t},
$$

and

$$
a_{i j}= \begin{cases}\left(\gamma-k \pi^{2}\right) \frac{(\gamma-\alpha)^{j}}{j !(\gamma+\alpha)^{j+1}} \alpha^{\frac{1}{2}} & (i=1), \\ 0 & (i \neq 1) .\end{cases}
$$

Hence, $v$ of (5) becomes,

$$
u(x, t)=\sin (\pi x) e^{-\gamma t}+\sum_{j=0}^{\infty}\left(\gamma-k \pi^{2}\right) \frac{(\gamma-\alpha)^{j}}{j !(\gamma+\alpha)^{j+1}} \alpha^{\frac{1}{2}} \phi_{1 j}(x, t) .
$$

If either $\gamma=k \pi^{2}$ or $\gamma=\alpha$, (16) becomes, using (13) for the second case,

$$
u(x, t)=\sin (\pi x) e^{-\pi^{2} t}
$$

which is the Fourier series solution of this initial boundary value problem.

In fact, the Example is a special case of

THEOREM 1. Let $H(x)$ be an odd periodic function, $H(2+x)=H(x)$, which vanishes at $x=-1,0$ and 1 , let its first derivative be absolutely continuous and its second derivative be contained in $L_{2}((-1,1))$. Then, for the initial boundary value problem defined by (3) and (4) with

$$
F(x)=H(x), g(t)=h(t)=0,0 \leq x \leq 1, t>0
$$

the variational representation (5) reduces to the Fourier series solution of (3), (4) and (17) when $\gamma=\alpha$.

Proof. Here,

$$
f(x, t)=\gamma H(x) e^{-\gamma t}+\kappa \frac{\partial^{2} H(x)}{\partial x^{2}} e^{-\gamma t} .
$$

Hence,

$$
a_{i j}=\frac{2(\gamma-\alpha)^{j} \alpha^{\frac{1}{2}}}{j !(\gamma+\alpha)^{j+1}}\left\{\gamma \int_{0}^{1} H(x) \sin (i \pi x) d x+\kappa \int_{0}^{1} \frac{\partial^{2} H(x)}{\partial x^{2}} \sin (i \pi x) d x\right\} .
$$

Let 


$$
b_{i}=2 \int_{0}^{1} H(x) \sin (i \pi x) d x \quad(i=1,2, \ldots)
$$

denote the Fourier coefficients of $H(x)$. Using (18) and the result of Zygmund [7, Chapter 2, 52] on the differentiation of Fourier series, one obtains for the variational representation (5) of the solution of this problem

$$
u(x, t)=H(x) e^{-\gamma t}+\sum_{i=1}^{\infty} \sum_{j=0}^{\infty} \frac{(\gamma-\alpha)^{j} \alpha^{\frac{1}{2}}}{j !(\gamma+\alpha)^{j+1}}\left(\gamma-\kappa i^{2} \pi^{2}\right) b_{i} \phi_{i j}(x, t) .
$$

Putting $\gamma=\alpha$ in this last expression and using (13), we obtain

$$
u(x, t)=H(x) e^{-\alpha t}-\sum_{i=1}^{\infty} b_{i} \sin (i \pi x) e^{-\alpha t}+\sum_{i=1}^{\infty} b_{i} \sin (i \pi x) e^{-\kappa i^{2} \pi^{2} t},
$$

since

$$
\phi_{i 0}=\frac{\sqrt{2} \sin (i \pi x)(2 \alpha)^{\frac{1}{2}}}{\left(\kappa i^{2} \pi^{2}-\alpha\right)}\left\{e^{-\alpha t}-e^{-\kappa i^{2} \pi^{2} t}\right\} \text {. }
$$

As a consequence of the conditions imposed on $H(x)$ in the theorem, the Fourier series of $H(x)$ converges uniformly to $H(x)$ (see [7, Chapter 2] or $[6, \S 13.25])$. Thus, the first two terms on the right hand side of (19) cancel and we obtain the Fourier series solution of (3), (4) and (19); which proves the theorem.

A more general result on the differentiation of Fourier series is LEMMA. Let the function $K(x)$ satisfy the following conditions:

(a) $K(x)$ is an odd function,

(b) $K(x)$ is periodic, $K(2+x)=K(x)$,

(c) $K(x)$ has discontinuities of the first kind (jumps) at $x=0$ and $x=1$, such that $K\left(0^{+}\right)=M_{1}, K\left(0^{-}\right)=-M_{1}$, $K\left(1^{+}\right)=-M_{2}, K\left(1^{-}\right)=M_{2}$, and

(d) the first derivative of $K(x)$ is absolutely contiruous on the intervals $(-1,0)$ and $(0,1)$ where $K^{\prime}(x)$ is completed by continuity at $x=-1,0$ and 1 .

Then, 


$$
S\left[K^{\prime \prime}(x)\right]=S^{\prime \prime}[K(x)]-2 \sum_{i=1}^{\infty}\left\{M_{2}(-1)^{i}-M_{1}\right\} i \pi \sin (i \pi x),
$$

where $S[f(x)]$ denotes the Fourier series of $f(x), S^{\prime \prime}[f(x)]$ its second derivative and $K^{\prime \prime}(x)=d^{2} K(x) / d x^{2}$.

The proof is straightforward. We only pause to note that, using (21),

$$
\begin{aligned}
\int_{0}^{1}\left\{\gamma\left[K(x)-(1-x) M_{1}-x M_{2}\right]+\kappa \frac{\partial^{2} K(x)}{\partial x^{2}}\right\} & \sin (i \pi x) d x \\
& =\frac{1}{2}\left(\gamma-\kappa i^{2} \pi^{2}\right)\left[d_{i}-2 \frac{M_{1}}{i \pi}+2(-1)^{i} \frac{M_{2}}{i \pi}\right],
\end{aligned}
$$

where

$$
d_{i}=\int_{-1}^{1} K(x) \sin (i \pi x) d x \quad(i=1,2, \ldots)
$$

Using (22), the following generalization of Theorem 1 is proved.

THEOREM 2. Let $H(x)$ be a function which satisfies the conditions of the lemma and is such that $H^{\prime \prime}(x)$ which is defined except at $-1,0$ and 1$\}$ is contained in $L_{2}((-1,1))$. Then, the solution of the initial boundary value problem defined by (3) and (4) with

(1) $F(x)=H(x)$, and

(2) $g(t)$ and $h(t)$ absolutely continuous and $g(0)=M_{1}$ and $h(0)=M_{2}$,

has the following representation

$$
\begin{aligned}
u(x, t)=(1-x) g(t)+x h(t)+\sum_{i=1}^{\infty} \sum_{j=0}^{\infty}\left[\frac { 2 \alpha ^ { \frac { 1 } { 2 } } } { j ! } \left\{-\frac{2}{i \pi}\left[(-1)^{i}-\frac{1}{2}\right] \times\right.\right. \\
\left.\left.\times \int_{0}^{\infty} \frac{\partial g}{\partial t} L_{j}(2 \alpha t) e^{-\alpha t} d t+\frac{(-1)^{i} \sqrt{2}}{i \pi} \int_{0}^{\infty} \frac{\partial h}{\partial t} L_{j}(2 \alpha t) e^{-\alpha t} d t\right\} \phi_{i j}(x, t)\right]+ \\
+\sum_{i=1}^{\infty} k_{i} \sin (i \pi x) e^{-k i^{2} \pi^{2} t},
\end{aligned}
$$

where the $k_{i}(i=1,2, \ldots)$ are the coefficients of the Fourier series of 
$f(x)=\left\{H(x)-(1-x) M_{1}-x M_{2}\right\}, \quad(0 \leq x \leq 1), f(-x)=f(x), f(x+2)=f(x)$.

Proof. Applying (22) to the variational representation (5) of the solution of initial boundary value problem of (3) and (4) formulated in the theorem, putting $\gamma=\alpha$ and using (18) and (20) as well as the facts that

$$
k_{i}=b_{i}-\frac{2 M_{1}}{i \pi}+\frac{2(-1)^{i} M_{2}}{i \pi} \quad(i=1,2, \ldots),
$$

and

$$
\sum_{i=1}^{\infty}\left(b_{i}-\frac{2 M_{i}}{i \pi}+2 \frac{(-1)^{i} M_{2}}{i \pi}\right) \sin (i \pi x)=H(x)-(1-x) M_{1}-x M_{2} \text {, }
$$

we obtain (23).

At least when $g(t)$ and $h(t)$ are constant with respect to $t$, (23) will be computationally more efficient than the corresponding solution found in Carslaw and Jaeger [2, 537, equation (2)].

\section{References}

[1] R.S. Anderssen, "A class of densely invertible parabolic operator equations", Bull. Austral. Math. Soc. 1 (1969), 363-374.

[2] H.S. Carslaw and J.C. Jaeger, Conduction of heat in solids (Clarendon Press, Oxford, 1947).

[3] A. Erdélyi, W. Magnus, F. Oberhettinger, F.G. Tricomi (edited by), Tables of integral transforms, Vol. 1. Based, in part, on notes left by Harry Bateman. (McGraw-Hill, New York, Toronto, London, 1954).

[4] S.G. Mikhlin, Variational methods in mathematical physics (translated by T. Boddington; Pergamon Press, Oxford; The Macmillan Co.,

[5] W.V. Petryshyn, "Direct and iterative methods for the solution of linear operator equations in Hilbert space", Trans. Amer. Math. Soc. 105 (1962), 136-175.

[6] E.C. Titchmarsh, The theory of functions, and ed., (Clarendon Press, Oxford, 1939). 
[7] A. Zygmund, Trigonometric series, Vol. 1, 2nd ed., (Cambridge University Press, Cambridge, 1959).

\section{Computer Centre,}

Australian National University,

Canberra, ACT. 